\title{
Polarization Properties of Specular and Dense Multipath Components in a Large Industrial Hall
}

\author{
Davy P. Gaillot, Emmeric Tanghe, Member, IEEE, Wout Joseph, Senior Member, IEEE, Pierre Laly, Viet-Chi \\ Tran, Martine Liénard, and Luc Martens, Member, IEEE
}

\begin{abstract}
This paper presents a comprehensive analysis of the polarization characteristics of specular and dense multipath components (SMC and DMC) in a large industrial hall based on frequency-domain channel sounding experiments at $1.3 \mathrm{GHz}$ with $22 \mathrm{MHz}$ bandwidth. 29 positions were measured under LineOf-Sight (LOS) and Obstructed LOS (OLOS) scenarios. The RiMAX maximum-likelihood estimator is used to extract the full-polarimetric SMC and DMC from the measurement data by taking into account the polarimetric radiating patterns of the dual-polarized antennas. Cross-polar discrimination (XPD) and co-polar ratio (CPR) values are presented from the measured and de-embedded channels as well as the polarimetric delay and angular spread distributions. Strong de-embedded SMC depolarization is obtained for the horizontal polarization in OLOS scenarios. Additionally, DMC depolarization is observed to be weaker than previously reported for indoor environments but constant across LOS/OLOS, polarization, and distance. The results also show that the co-polar (cross-polar) DMC power to total channel power ratio is equal to $15 \%(40 \%)$ for LOS and $40 \%(60 \%)$ for OLOS and that this ratio does not correlate significantly with transmitter-receiver distance. Finally, the validity of the room electromagnetics theory was confirmed for transmitterreceiver distances larger than $\mathbf{1 5} \mathbf{m}$ with no significant difference between polarized subchannels.
\end{abstract}

Index Terms-MIMO systems, indoor propagation, maximum likelihood estimation.

\section{INTRODUCTION}

$\mathbf{O}$ VER the course of the last decade, the physical view of the radio channel has undergone an important change. Before that, the radio channel was commonly considered to be but a collection of specular multipath components (SMC) that have well-defined discrete locations in the different radio channel dimensions (e.g., space, frequency, time, etc.). Nowadays, it is widely accepted that part of the radio channel is also continuous across these dimensions. This part is put under the umbrella of dense multipath components (DMC). Among other sources, DMC originates from distributed diffuse scattering on electrically small objects and is typically observed as the decay slope of the power delay profile [1]. DMC models for indoor environments [2], [3], [4], [5], [6], [7] rely on the room electromagnetics theory for diffuse scattering observed in reverberation chambers [8], [9]. In this theory, the reverberation

D. P. Gaillot, P. Laly, and M. Liénard are with the University of Lille I, IEMN, Bâtiment P3,Villeneuve d'Ascq, 59655 FR e-mail: davy.gaillot@univlille1.fr.

V.-C. Tran is with the University of Lille I, Department of Mathematics, Villeneuve d'Ascq, 59655.

E. Tanghe, L. Martens, and W. Joseph are with Ghent University/iMinds, Department of Information Technology, B-9050 Ghent, Belgium, e-mail: emmeric.tanghe@intec.ugent.be.

Manuscript received July xx, 2014; revised xx xx, 2014. components power density is described as an exponentially decaying function of delay. More recently, a distance dependent model was reported for indoor radio channels where the power delay profile is described as the sum of primary SMC and DMC [10]. Naturally, the introduction of DMC in the physical model of the radio channel means that common radio channel parameters have to be re-evaluated for dense multipath as well. Moreover, contemporary radio channel models are expected to encompass a complete polarimetric description of the physical radio link. However, a joint polarimetric analysis of SMC and $\mathrm{DMC}$ in indoor or related scenarios is missing in the literature to the authors' knowledge.

This contribution fits within this effort: we present for the first time a comprehensive analysis of the SMC and DMC cross-polarization discrimination (XPD) and co-polar ratio (CPR) parameters in a high-volume industrial environment. The analysis is based on frequency-domain channel sounding experiments at $1.3 \mathrm{GHz}$ with $22 \mathrm{MHz}$ bandwidth. The RiMAX maximum-likelihood estimator is used to extract the full-polarimetric specular and dense multipath components from the channel sounding data [1]. Section II presents the measurement environment, experimental procedure, as well as the data processing. A detailed polarimetric analysis of the measured and de-embedded channels is presented in Section III. Before concluding, an analysis of the DMC reverberation time is presented and the validity of the room electromagnetics theory is discussed for this environment. In this work, matrices (vectors) are denoted with upper (lower) case boldface letters (e.g. A, or a).

\section{Measurements And Data Processing}

\section{A. Measurement environment and procedure}

The propagation environment under consideration is a large industrial hall located in Zwijnaarde, Belgium. The hall has dimensions $21.3 \times 77.2 \times 12.2 \mathrm{~m}^{3}$ and is dedicated to the research of concrete technology. The main inventory consists of large metallic machinery used to test the robustness of small to large concrete structures. The dominant building material for walls, floor, and ceiling is concrete. The windows are located near the ceiling and a large metallic industrial door closes off one end of the hall. Figure 1(a) presents a view of the industrial hall.

Multidimensional channel sounding measurements were performed in the industrial hall. A 4-port vector network analyzer (VNA) of reference Agilent E5071C was used to probe the full polarimetric radio channel in a $22 \mathrm{MHz}$ bandwidth centered 
around $1.3 \mathrm{GHz}$ in $M_{f}=1601$ uniformly spaced frequency points. This central frequency was selected to describe the physical propagation phenomena for the mobile and WiFI standards without emitting in the licenced bands. At both link ends, a virtual antenna array was created by an antenna mounted on an automated rotating arm. At both transmit (Tx) and receive $(\mathrm{Rx})$ side, dual-polarized antennas were used, installed at $1.60 \mathrm{~m}$ above ground level. The virtual array was a planar horizontal uniform circular array (UCA) with radius $15 \mathrm{~cm}$ and consisting of $M_{T}=M_{R}=12$ antenna elements. A $500 \mathrm{~m}$ optical fibre was deployed for the transmitting side with a RF to Optics / Optics to RF interface to allow the Tx to move within a $500 \mathrm{~m}$ radius of the Rx. The 4-port VNA allows measuring simultaneously the Vertical $(V)$ and Horizontal $(H)$ components of Rx. A $50 \mathrm{~dB}$ isolation switch was placed behind Tx to manually switch between $H$ and $V$. The system was through calibrated to remove the attenuation introduced by the optical system setup and the RF cables. In addition, the VNA power was set to $10 \mathrm{dBm}$ and an additional $1 \mathrm{~W}$ power amplifier was placed at the transmitting side to increase the receiver SNR. The dual-polarized antennas for Tx and Rx are identical $1.6 \mathrm{~mm}$ thick FR4 $\left(\epsilon_{r}=4.35\right)$ square patches. The size of the copper ground plane is $11.06 \mathrm{~cm} \times 11.06 \mathrm{~cm}$ and $55.9 \mathrm{~mm} \times 55.9 \mathrm{~mm}$ for the active region. Via-hole SMA feeds were positioned horizontally and vertically at $1.575 \mathrm{~cm}$ from the center of the active region to obtain orthogonal modes between $V$ and $H$ with $50 \Omega$ characteristic impedance. Both antennas display a $22 \mathrm{MHz}$ bandwidth measured for a return loss lower than -10 dB. Figure 2 presents the measured co- and cross-polar far-field radiating patterns for the $H$ and $V$ ports at $1.3 \mathrm{GHz}$. As shown, the antennas have a minimum $15 \mathrm{~dB}$ XPD with computed $120^{\circ}$ and $100^{\circ}$ beamwidth at half power measured in the azimuth and elevation plane, respectively.

Figure 1(b) depicts the 29 measurement locations considered in this work. The selected scenarios were either Line-Of-Sight (LOS) or Obstructed Line-Of-Sight (OLOS). For 26 links, Tx was moved around the hall whereas $\mathrm{Rx}$ was fixed. For the 3 remaining links, Tx and Rx were both moved. The locations of the Tx (Rx) are shown as blue circles (red squares). Tx and $\mathrm{Rx}$ locations sharing the same number form a Tx - Rx measurement pair. In addition, the black squares represent the tall concrete supports at each side of the hall.

\section{B. Specular and Dense Multipath Component Estimation}

Typically, the full polarimetric channel matrix is given by:

$$
\mathbf{H}=\left(\begin{array}{cc}
\mathbf{h}_{H H} & \mathbf{h}_{H V} \\
\mathbf{h}_{V H} & \mathbf{h}_{V V}
\end{array}\right),
$$

where the sampled array response vector $\mathbf{h}_{X Y} \in$ $\mathbb{C}^{M_{R} M_{T} M_{f} \times 1}$ can be written as the sum of an SMC part $\mathbf{s}_{X Y}$ and a DMC part $\mathbf{d}_{X Y}$. The subscripts $X$ and $Y$ denote the polarization of the transmitting and receiving antenna, respectively. $X$ and $Y$ are either horizontal $(H)$ or vertical $(V)$. It is commonly assumed that $\mathbf{h}_{X Y}$ follows a multivariate circularly symmetric complex Gaussian distribution [1]:

$$
\mathbf{h}_{X Y}=\mathbf{s}_{X Y}\left(\boldsymbol{\theta}_{s}^{X Y}\right)+\mathbf{d}_{X Y}\left(\boldsymbol{\theta}_{d}^{X Y}\right)
$$

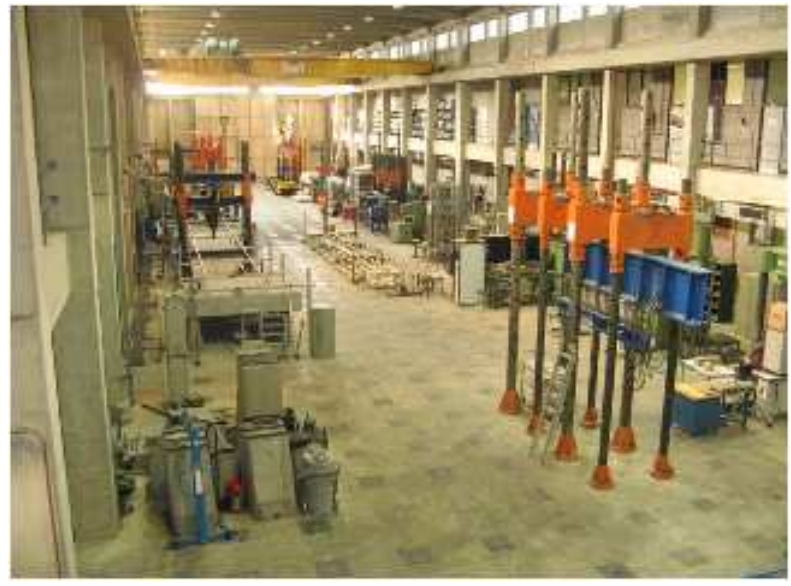

(a)

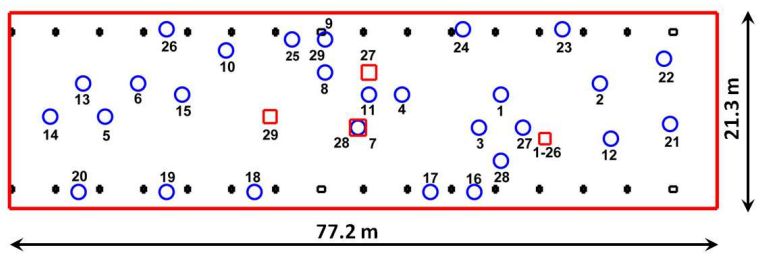

(b)

Fig. 1. Measurement environment. (a) Industrial hall. (b) Tx (circles) - Rx (squares) measurement pairs. The black squares indicate the position of the tall concrete supports at each side of the hall.

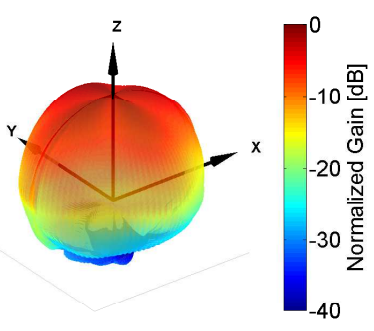

(a)

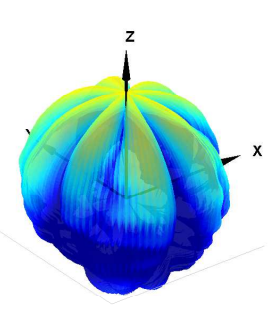

(c)

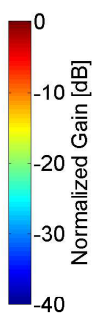

0
Fig. 2. Normalized far-field radiating patterns for $V V$ (a), $V H$ (b), $H V$ (c), and $H H(\mathrm{~d})$ (in $\mathrm{dB}$ ) measured at $1.3 \mathrm{GHz}$. The patterns have been normalized with respect to the maximum gain for each port. 


$$
\mathbf{h}_{X Y} \sim \mathcal{N}_{c}\left(\mathbf{s}_{X Y}\left(\boldsymbol{\theta}_{s}^{X Y}\right), \mathbf{R}_{X Y}\left(\boldsymbol{\theta}_{d}^{X Y}\right)\right) .
$$

The parameter vector $\boldsymbol{\theta}_{s}^{X Y}$ groups the parameters associated with the SMCs. On one hand, these include the geometrical parameters that define each specular path in the propagation environment: the angles of arrival/departure and the time-delay of arrival. The geometrical SMC parameters are identical across all four polarization subchannels $X Y$. On the other hand, $\boldsymbol{\theta}_{s}^{X Y}$ also includes the complex amplitude $\gamma_{X Y}$ of the SMCs. The complex amplitude will differ between different polarization subchannels because of the polarization-dependency of electromagnetic interactions (reflections, diffractions, etc.).

The DMC part $\mathbf{d}_{X Y}\left(\boldsymbol{\theta}_{d}^{X Y}\right)$ is fully determined by the channel covariance matrix. The covariance matrix is assumed to have the following structure involving Kronecker products [1]:

$$
\mathbf{R}_{X Y}\left(\boldsymbol{\theta}_{d}^{X Y}\right)=\mathbf{I}_{M_{R}} \otimes \mathbf{I}_{M_{T}} \otimes \mathbf{R}_{f}^{X Y}\left(\alpha_{0}^{X Y}, \alpha_{1}^{X Y}, \beta_{d}^{X Y}, \tau_{d}^{X Y}\right) .
$$

In (4), I represents the identity matrix. The dense multipath field is modeled as uncorrelated in the spatial (angular) domains ( $\mathbf{I}_{M_{R}}$ and $\mathbf{I}_{M_{T}}$ ) but correlated in the frequency (timedelay) domain $\left(\mathbf{R}_{f}^{X Y}\right)$. The DMC power delay profile as a function of time-delay $\psi_{X Y}(\tau)$ is described by an exponential decay:

$$
\psi_{X Y}(\tau)=\alpha_{1}^{X Y} e^{-\beta_{d}^{X Y}\left(\tau-\tau_{d}^{X Y}\right)}+\alpha_{0}^{X Y} .
$$

In (4) and (5), $\alpha_{1}^{X Y}, \beta_{d}^{X Y}, \tau_{d}^{X Y}$, and $\alpha_{0}^{X Y}$ are four parameters which fully describe the DMC of polarization subchannel $X Y$ and are gathered into the DMC parameter vector $\boldsymbol{\theta}_{d}^{X Y}$. Deterministic and stochastic maximum-likelihood estimators of $\boldsymbol{\theta}_{s}^{X Y}$ and $\boldsymbol{\theta}_{d}^{X Y}$, respectively, are obtained from the channel sounding data using the iterative multipath estimation algorithm RiMAX [1]. To this end, the de-embedding of the fullpolarimetric complex radiation patterns of the measurement antennas was performed to assess the full polarimetric complex gains for each path. Our approach is based on the concept of the Effective Aperture Distribution Function (EADF) detailed in [11].

\section{Polarimetric rms Delay Spread Characteristics}

The root-mean-square (rms) delay spread relates to the maximum data rate without inter-symbol interference (ISI) and is, therefore, one of the key characteristics when designing wireless wideband communication systems. It is calculated as the square root of the second central moment of the Power Delay Profile (PDP) [12]. Here, the PDP is obtained from each polarimetric channel matrix (1) and averaged over all $\mathrm{Tx}$ - Rx links to remove the small-scale fading:

$$
\operatorname{PDP}_{X Y}(\tau)=\frac{1}{M_{T} M_{R}} \sum_{m=1}^{M_{T}} \sum_{n=1}^{M_{R}}\left|\mathcal{F}^{-1}\left(\mathbf{h}_{X Y}(f, m, n)\right)\right|^{2},
$$

where $\tau$ is the time-delay, $f$ the sampled frequency, and $\mathcal{F}^{-1}$ the inverse Fourier operator. As an example, Fig. 3 presents the measured polarimetric PDP for a LOS (position 15) and OLOS (position 18) shadowing scenario. The exponential decay of the PDP indicates the presence of DMC for both scenarios and all polarization links. In addition, the strong peaks in the PDP shape are attributed to the SMC but cannot be distinguished from each other due to the limited bandwidth. The polarimetric rms delay spread $\tau_{r m s}^{X Y}$ (in ns) is computed from (6) and is given by:

$\tau_{r m s}^{X Y}=\sqrt{\frac{\sum_{p} \mathbf{P D P}_{X Y}\left(\tau_{p}\right)\left(\tau_{p}\right)^{2}}{\sum_{p} \mathbf{P D P}_{X Y}}-\left(\frac{\sum_{p} \mathbf{P D P}_{X Y}\left(\tau_{p}\right)\left(\tau_{p}\right)}{\sum_{p} \mathbf{P D P}_{X Y}}\right)}$.

A $20 \mathrm{~dB}$ threshold from the peak of the PDP was selected for the computation of $\tau_{r m s}^{X Y}$. Note that the difference between the peak and thermal noise was greater than $35 \mathrm{~dB}$ for all polarimetric PDP measured for this work. Figure 4 presents the Cumulative Distribution Function (CDF) of $\tau_{r m s}^{X Y}$ for the LOS and OLOS scenarios. In addition, Table I presents the $\tau_{r m s}^{X Y}$ values averaged over all positions for the LOS and OLOS scenarios. Firstly, it is noted that the observed spreads are within the range of values found in indoor scenarios at this frequency [12]. The delay spread values are less than $65 \mathrm{~ns}$ and $70 \mathrm{~ns}$ for LOS and OLOS, respectively. However, it is observed that the LOS co-polar values are more spread out compared to any other scenarios or polarization resulting in a smaller averaged rms delay spread of $35 \mathrm{~ns}$. This is attributed to the fact that SMC are rather strong for the LOS co-polar scenarios. The same observation is made at $11 \mathrm{GHz}$ for officetype environments in [13]. This finding will be discussed in the next section.

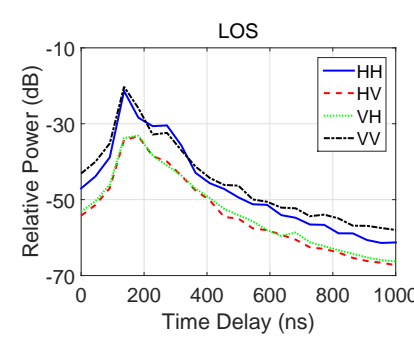

(a)

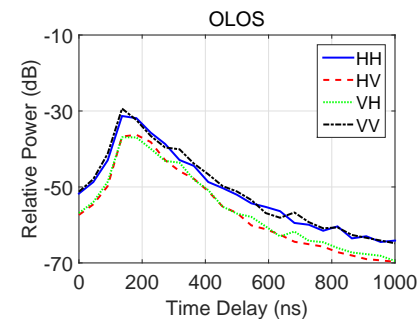

(b)
Fig. 3. Example of measured polarimetric PDP for a LOS (position 15) (a) and OLOS (position 18) (b) shadowing scenario.

TABLE I

Averaged PolarimetriC RMS DELAy SPREAD (IN $n s$ )

\begin{tabular}{|c|c|c|c|c|}
\cline { 2 - 5 } \multicolumn{1}{c|}{} & $\tau_{r m s}^{H H}$ & $\tau_{r m s}^{H V}$ & $\tau_{r m s}^{V H}$ & $\tau_{r m s}^{V V}$ \\
\hline \hline LOS & 35 & 52.5 & 51.8 & 35.5 \\
\hline OLOS & 50.2 & 56.2 & 56.7 & 50.2 \\
\hline
\end{tabular}

\section{RESULTS}

Since the measured polarimetric channels include both the contribution of the propagation (i.e. SMC and DMC) and 


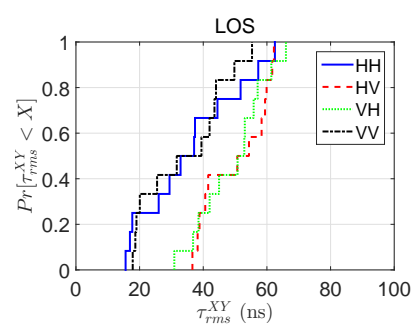

(a)

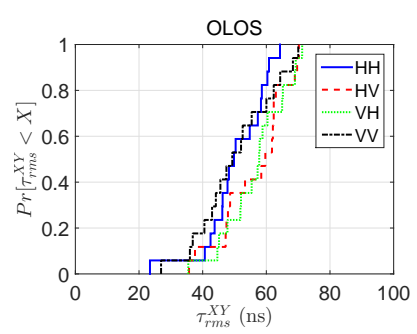

(b)
Fig. 4. Cumulative Distribution Function (CDF) of $\tau_{r m s}^{X Y}$ (in ns) for the LOS (a) and OLOS (b) shadowing scenarios.

transmission channel (i.e. antennas), the physical interpretation of the propagation phenomena might be rather different or misleading if the polarimetric SMC/DMC and antenna effects are included or de-embedded. A comparison between both approaches is needed as the former relates to general propagation effects typically discussed in the litterature (III-B) whereas the latter provides a deeper comprehension of those effects (III-C).

\section{A. Estimated Measured Power}

In this subsection, the total measured power $P_{T}$ as well as the estimated SMC and DMC power $\left(P_{S M C}\right.$ and $\left.P_{D M C}\right)$ are investigated as a function of polarization, shadowing condition, and Tx-Rx distance. Both SMC and DMC were initially reconstructed over the $22 \mathrm{MHz}$ bandwidth. Also, the small-scale effects were separated from the large-scale effects by averaging, for each position, the received power of the 144 spatial subchannels. Figure 5 presents the LOS (left column) and OLOS (right column) data for all polarization subchannels. The results indicate that the power is decreasing with distance but that it also fades more rapidly for OLOS scenarios. The estimated path loss exponent for LOS scenarios varies between $1.7(H V / V H)$ and $1.8(H H / V V)$, whereas values above 2 were found for OLOS scenarios (2.2 for $H V / V H$ and 2.65 for $H H / V V$ ) in agreement with values reported in [12]. For the co-polar links, the contribution of the SMC to the total power is larger for LOS ((a) and $(\mathrm{g})$ ) than OLOS ((b) and (h)) due to the LOS and primary specular components (single-order reflections) [10]. However, the SMC and DMC power are closer to each other for the cross-polar links $H V$ (c) and $V H$ (e) under LOS scenarios. This effect is attributed to the depolarization mechanisms for the higherorder specular components. This is confirmed by the fact that the DMC power becomes stronger than the SMC for OLOS scenarios since the LOS and primary specular components are missing or strongly attenuated. It is noteworthy that the SMC and DMC power show on average a strong correlation of 0.90 (LOS) and 0.95 (OLOS). This implies the DMC can alternatively be interpreted as the non-coherent superposition of paths with weaker signal-to-noise ratios (SNR) which still follow the specular power decay as a function of distance [14], [15].

In addition, Fig. 6 presents the DMC to total power ratio $P_{D M C} / P_{T}$ (in \%) for each polarization link as a function of distance. The results indicate that the normalized DMC

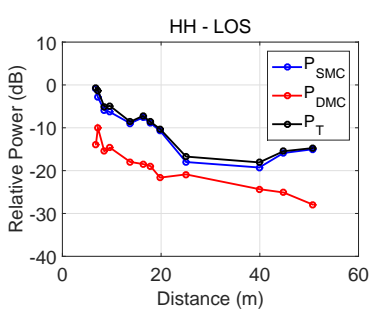

(a)

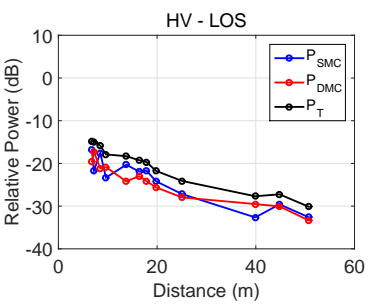

(c)

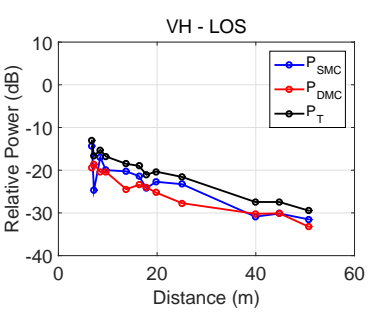

(e)

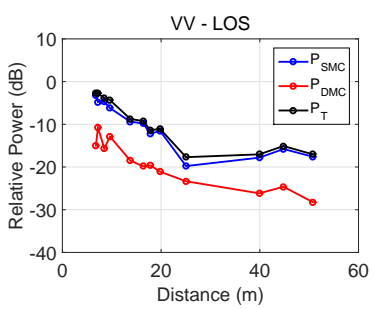

(g)

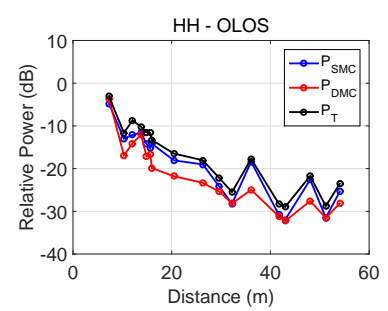

(b)

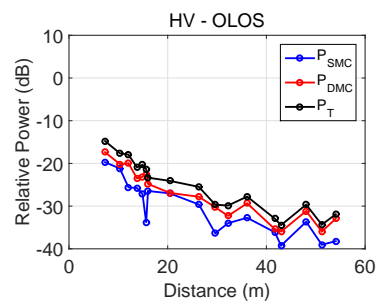

(d)

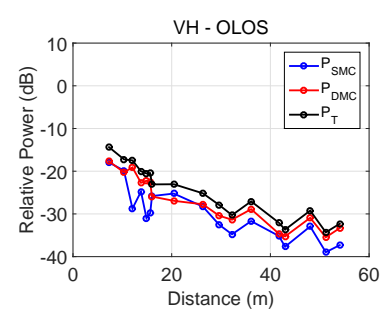

(f)

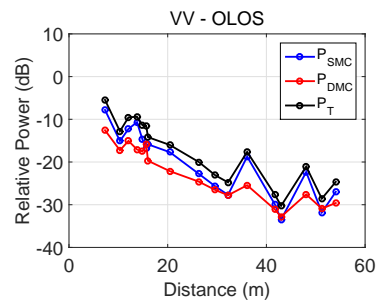

(h)
Fig. 5. $P_{T}, P_{S P}$, and $P_{D M C}$ as a function of distance (in $\mathrm{m}$ ) for $H H$ (1 ${ }^{\text {st }}$ row), $H V$ ( $2^{\text {nd }}$ row), $V H$ ( $3^{\text {rd }}$ row), and $V V$ ( $4^{\text {th }}$ row), and for LOS (left column) and OLOS (right column).

power is strongly dependent on the link shadowing [16] but also on the polarization. Conversely, the normalized DMC power does not appear to strongly correlate with Tx-Rx distance: this is confirmed by permutation hypothesis tests, which concluded that Spearman's rho correlation coefficient does not differ significantly from zero (calculated on the normalized DMC power of all four polarization subchannels combined: $p$-values of 0.48 and 0.61 were obtained for LOS and OLOS, respectively). For the smallest OLOS distance, it is noticed a larger DMC power ratio for $H H$ compared to $V V$. This difference is attributed to the presence of horizontal and vertical concrete slabs located half a meter away from the Tx antenna array which partially obstruct the LOS. Here, this effect is believed to be attributed to the Brewster angle for the $H H$ polarization as also reported in [13]. The most dominant SMC will be highly attenuated if they present low elevation angles and undergo grazing reflections with large angles of 


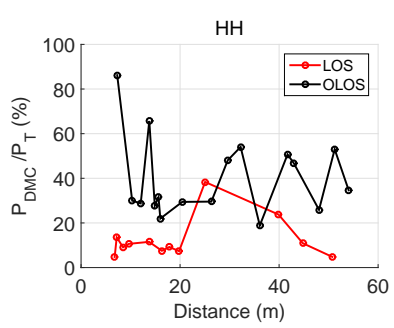

(a)

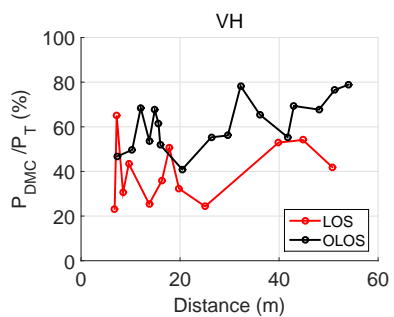

(c)

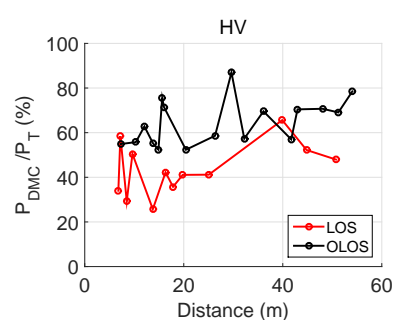

(b)

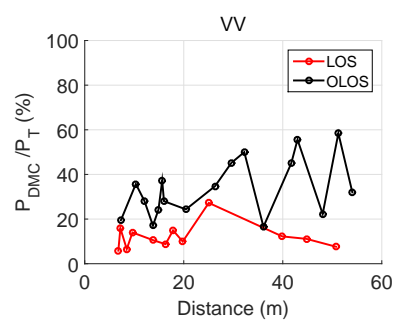

(d)
Fig. 6. $P_{D M C} / P_{T}$ (in \%) as a function of distance (in $\mathrm{m}$ ) for $H H$ (a), $H V$ (b), $V H$ (c), and $V V$ (d), and for LOS (red) and OLOS (black) scenarios.

incidence. In contrast, the DMC is presumably not affected by this as this mechanism is likely more isotropic in nature and not clustered around the Brewster angle.

The mean values $\hat{\mu}$ and standard deviations $\hat{\sigma}$ of the normalized DMC power are presented in Table II for each polarization subchannel and link shadowing condition. For the co-polar links, the results are found to be in agreement with recently reported values for indoor [17] and industrial environments [18]. The contribution of DMC to the total channel power is noticeably larger for the cross-polar links when compared to the co-polar links. For OLOS, the crosspolar normalized DMC power on average even exceeds $60 \%$, indicating that these channels could be modelled relatively accurately by only considering their DMC (as it is done in the original room electromagnetics model [4], [6]).

TABLE II

$P_{D M C} / P_{T}($ IN $\%)$

\begin{tabular}{c|c|c|c|c|c|c|c|c|}
\cline { 2 - 10 } & \multicolumn{2}{|c|}{$H H$} & \multicolumn{2}{c|}{$H V$} & \multicolumn{2}{c|}{$V H$} & \multicolumn{2}{c|}{$V V$} \\
\cline { 2 - 10 } & $\hat{\mu}$ & $\widehat{\sigma}$ & $\hat{\mu}$ & $\widehat{\sigma}$ & $\hat{\mu}$ & $\widehat{\sigma}$ & $\hat{\mu}$ & $\widehat{\sigma}$ \\
\hline \hline LOS & 12.6 & 9.5 & 43.6 & 11.8 & 40 & 13.6 & 12 & 5.8 \\
\hline OLOS & 40.6 & 17.8 & 64.6 & 10.2 & 61.4 & 11.3 & 33.8 & 13.2 \\
\hline
\end{tabular}

\section{B. Measured XPD and CPR}

Typically, the XPD and CPR values are studied directly from the measured polarimetric transfer functions or indirectly from the PDPs computed from (6). However, this approach suffers two major drawbacks. First, the polarimetric contribution of the SMC and DMC to the radio channel cannot be assessed. Also, since the antenna effects are included, it follows that the description of the polarization mechanisms via the XPD or CPR is only meaningful for the case where omnidirectional antennas are being used for the measurement.
Directive antennas are classically used in polarimetric channel sounding systems and this work falls into this scheme. Hence, the purpose of this study is to highlight the fact that the analysis of the XPD/CPR from the measured channel might be incorrectly interpreted when the polarimetric patterns of the virtual array is not de-embedded.

The XPD for $H$ and $V$ as well as the CPR are given for each position by:

$$
\begin{gathered}
\mathbf{X P D}_{H}(d B)=10 \log _{10}\left(\frac{\sum_{p} \mathbf{P D P}_{H H}\left(\tau_{p}\right)}{\sum_{p} \mathbf{P D P}_{H V}\left(\tau_{p}\right)}\right), \\
\mathbf{X P D}_{V}(d B)=10 \log _{10}\left(\frac{\sum_{p} \mathbf{P D P}_{V V}\left(\tau_{p}\right)}{\sum_{p} \mathbf{P D P}_{V H}\left(\tau_{p}\right)}\right), \\
\mathbf{C P R}(d B)=10 \log _{10}\left(\frac{\sum_{p} \mathbf{P D P}_{H H}\left(\tau_{p}\right)}{\sum_{p} \mathbf{P D P}_{V V}\left(\tau_{p}\right)}\right) .
\end{gathered}
$$

Table III presents the averaged $\left\langle\mathrm{XPD}_{H / V}\right\rangle$ and $\langle\mathrm{CPR}\rangle$ values estimated from the measured LOS and OLOS scenarios. In spite of the fact that the reported XPD values are close to those found in the literature for indoor environments [19], [20], [21], it is noted that there is slightly more depolarization with $V$ than $H$. This is attributed to the $1 \mathrm{~dB}$ gain difference measured between the $H$ and $V$ ports of our patch antennas and confirmed by the averaged CPR values for both LOS and OLOS.

TABLE III

Measured Averaged XPD \& CPR Values (IN $d B$ )

\begin{tabular}{|c|c|c|c|}
\cline { 2 - 4 } \multicolumn{1}{c|}{} & $\left\langle\mathrm{XPD}_{H}\right\rangle$ & $\left\langle\mathrm{XPD}_{V}\right\rangle$ & $\langle\mathrm{CPR}\rangle$ \\
\hline \hline LOS & 12.2 & 11.05 & 0.97 \\
\hline OLOS & 8.48 & 7.58 & 0.5 \\
\hline
\end{tabular}

The measured XPD and CPR results were compared with those presented in [13] at $11 \mathrm{GHz}$ in office-type environments. For a fair comparison, only the measurements in [13] that were taken in LOS in large halls (an entrance hall and an event hall) of similar size as our industrial hall were considered. For these halls, the measured LOS XPD values range between 10 and 21 $\mathrm{dB}$. The average LOS XPDs in Table III (11-12 dB) are on the low end of those reported and in line with their observation that XPD appears to increase with frequency due to the increased scattering losses in the cross-polar transmissions. The metallic, highly scattering inventory of our industrial hall does not seem to drastically alter the power distribution between the measured co- and cross-polar transmissions. Furthermore, the CPR values in [13] range between -6 and $7 \mathrm{~dB}$ : this is consistent with our average CPR value of $1 \mathrm{~dB}$ in Table III.

\section{De-embedded SMC and DMC}

In this subsection, the SMC and DMC are de-embedded from the measured channels with RiMAX by taking into account the measurement antennas. RiMAX is an iterative algorithm wherein a fixed number of SMC are estimated for each iteration step along with the DMC. Here, the number of new SMC per iteration was set to 5 as originally suggested 
in [1]. 50 iterations were chosen such that the maximum number of SMC was potentially 250. The reliability of each new SMC is checked with an SNR criterion [1], [18]. The algorithm stops itself when all 5 SMC fail the criterion.

The mean number of estimated paths per position was found to be around 139 and 85 for LOS and OLOS scenarios, respectively. Physically, this difference can be attributed to the fading mechanisms of the OLOS scenarios. For those scenarios, the SMC with longer delays would exhibit lower SNR and directly fall under the DMC umbrella since the DMC power ratio was shown to be strong. Consequently, it is expected that the number of estimated SMC is lower than for LOS scenarios.

1) Polarimetric rms Angular Spread Characteristics: For each position, the angle of arrival and departure were estimated for each path $p$. Since a horizontal UCA was used at both Tx and Rx, the estimated elevation angles were considered not sufficiently accurate and were discarded for the analysis. The angles of arrival $\left(\varphi_{R_{X}}^{A z, p}\right)$ and departure $\left(\varphi_{T_{X}}^{A z, p}\right)$ in azimuth are observed to be widely spread out which is attributed to the complexity of the scenario (highly metallic, very large number of metallic scatterers, etc.). A deeper understanding of the double-directional properties of the channel is provided by the statistical distribution of the rms angular spread $\varphi_{r m s}^{Z-X Y}$ where $Z$ is either Tx or Rx. Similarly to the polarimetric rms delay spread, a $20 \mathrm{~dB}$ threshold was used to compute the polarimetric $\varphi_{\mathrm{rms}}^{Z-X Y}$ given by:

$\varphi_{r m s}^{Z-X Y}=\sqrt{\frac{\sum_{p}\left|\gamma_{X Y}^{p}\right|^{2}\left(\varphi_{Z}^{A z, p}\right)^{2}}{\sum_{p}\left|\gamma_{X Y}^{p}\right|^{2}}-\left(\frac{\sum_{p}\left|\gamma_{X Y}^{p}\right|^{2}\left(\varphi_{Z}^{A z, p}\right)}{\sum_{p}\left|\gamma_{X Y}^{p}\right|^{2}}\right)}$

Figure 7 presents the CDF of the polarimetric rms angular spread for $\operatorname{Rx}\left(\varphi_{r m s}^{R_{X}-X Y}\right)$ and $\operatorname{Tx}\left(\varphi_{r m s}^{T_{X}-X Y}\right)$ for the LOS and OLOS scenarios. In addition, Table IV presents the $\varphi_{r m s}^{Z-X Y}$ values averaged over all positions for the LOS and OLOS scenarios. The data indicate that $\varphi_{r m s}^{R_{X}-X Y}$ are more spread out on average for LOS and OLOS scenarios ((a) and (b)) compared to $\varphi_{\text {rms }}^{T_{X}-X Y}$ ((c) and (d)), respectively. In addition, the LOS spread values are found to be in-between the minimum and maximum OLOS values. These mechanisms are believed to be attributed to the dense distribution of scatterers or equivalently to the low (high) scattering mean free path around the Tx (Rx) position. For instance, Tx was always located behind or nearby metallic equipment/machinery and concrete materials whereas $\mathrm{Rx}$ was located in a more open volume with few scatterers around. In other words, the receiving aperture is expected to be much larger than the emitting one. Consequently, the probability of receiving paths from a wider collection angle is also expected. Obviously, this effect is amplified when OLOS scenarios are considered (i.e. larger $\varphi_{r m s}^{R_{X}-X Y}$ and lower $\varphi_{r m s}^{T_{X}-X Y}$ values). Furthermore, it is observed that the spread values are uniform across polarization links for OLOS indicating that the depolarization mechanisms are similar for those scenarios. In contrast, the presence of weakly depolarized SMC in the LOS scenarios (LOS and firstorder paths) contribute to obtaining non-uniform polarimetric spread values.

Finally, we note that a clustering-based polarimetric channel model could be developed from the de-embedded data. However, the development of such a complete channel model would require separate extensive studies and is outside the scope of this paper. Indeed, this paper primarily focuses on the XPD/CPR characteristics of the SMC and DMC for the investigated scenario.

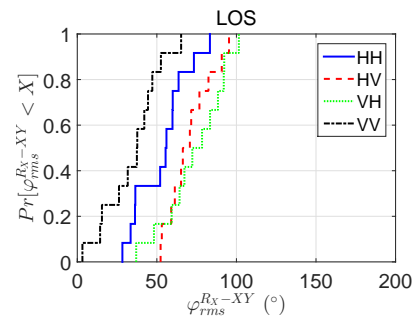

(a)

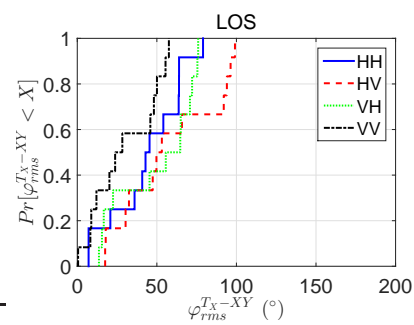

(c)

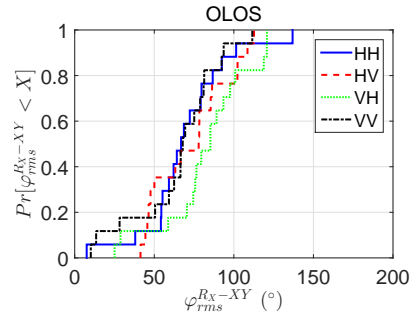

(b)

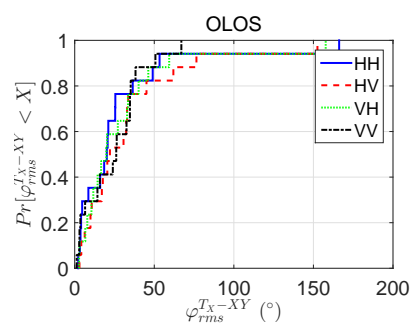

(d)
Fig. 7. $\mathrm{CDF}\left(\right.$ in ${ }^{\circ}$ ) of $\varphi_{r m s}^{R_{X}-X Y}$ (top row) and $\varphi_{r m s}^{T_{X}-X Y}$ (bottom row) for the LOS (left column) and OLOS (right column) shadowing scenarios.

TABLE IV

Averaged Polarimetric RMS Angular Spread ( IN $\left.^{\circ}\right)$

\begin{tabular}{|c|c|c|c|c|c|}
\cline { 3 - 6 } \multicolumn{2}{c|}{} & $H H$ & $H V$ & $V H$ & $V V$ \\
\hline \hline \multirow{2}{*}{ LOS } & $\varphi_{r m s}^{R_{X}}$ & 53.2 & 70.4 & 73.7 & 34.8 \\
\cline { 2 - 6 } & $\varphi_{r m s}^{T_{X}}$ & 43.7 & 58 & 49.4 & 29.9 \\
\hline \multirow{2}{*}{ OLOS } & $\varphi_{r m s}^{R_{X}}$ & 69.3 & 72.8 & 82.4 & 65.2 \\
\cline { 2 - 6 } & $\varphi_{r m s}^{T_{X}}$ & 28.1 & 33.9 & 30 & 24.5 \\
\hline
\end{tabular}

2) De-embedded XPD and CPR: The de-embedded SMC $\mathrm{XPD}$ and CPR were investigated per estimated path $p$ for each position. It is noteworthy that all estimated paths were considered for this study. The XPD and CPR per path are given by:

$$
\begin{aligned}
& \operatorname{XPD}_{H}^{p}(d B)=10 \log _{10}\left(\frac{\left|\gamma_{H H}^{p}\right|^{2}}{\left|\gamma_{H V}^{p}\right|^{2}}\right), \\
& \operatorname{XPD}_{V}^{p}(d B)=10 \log _{10}\left(\frac{\left|\gamma_{V V}^{p}\right|^{2}}{\left|\gamma_{V H}^{p}\right|^{2}}\right), \\
& \operatorname{CPR}^{p}(d B)=10 \log _{10}\left(\frac{\left|\gamma_{H H}^{p}\right|^{2}}{\left|\gamma_{V V}^{p}\right|^{2}} \cdot\right) .
\end{aligned}
$$




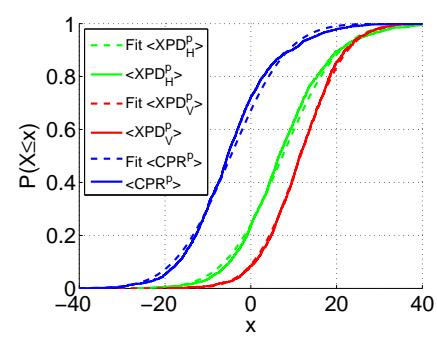

(a)

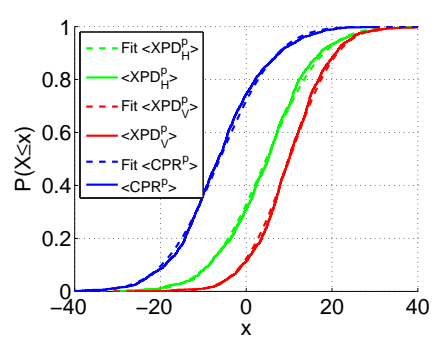

(b)
Fig. 8. CDF of $\left\langle\mathrm{XPD}_{H}^{p}\right\rangle,\left\langle\mathrm{XPD}_{V}^{p}\right\rangle$, and $\left\langle\mathrm{CPR}^{p}\right\rangle$, for LOS (a) and OLOS (b) scenarios.

The Cumulative Distribution Function (CDF) of $\left\langle\mathrm{XPD}_{H}^{p}\right\rangle$, $\left\langle\mathrm{XPD}_{V}^{p}\right\rangle$, and $\left\langle\mathrm{CPR}^{p}\right\rangle$ are presented in Fig. 8 for LOS and OLOS scenarios. First, one observes that the LOS and OLOS distributions show an identical behaviour. However, larger XPD values are observed for LOS scenarios since the loworder components are less affected by de-polarization mechanisms. It was also found that the distributions can be well fitted by normal distributions. Table $\mathrm{V}$ presents the fitted normal distribution parameters $\hat{\mu}$ and $\widehat{\sigma^{2}}$.

TABLE V

FitTed Normal Distribution PARAMETERs FOR SMC (IN $d B$ ).

\begin{tabular}{c|c|c|c|c|c|c|}
\cline { 2 - 7 } \multicolumn{1}{c|}{} & \multicolumn{2}{c|}{$\mathrm{XPD}_{H}^{p}$} & \multicolumn{2}{c|}{$\mathrm{XPD}_{V}^{p}$} & \multicolumn{2}{c|}{$\mathrm{CPR}^{p}$} \\
\cline { 2 - 7 } & $\hat{\mu}$ & $\widehat{\sigma^{2}}$ & $\hat{\mu}$ & $\widehat{\sigma^{2}}$ & $\hat{\mu}$ & $\widehat{\sigma^{2}}$ \\
\hline \hline LOS & 7.41 & 10.58 & 11.6 & 8.62 & -4.65 & 10.5 \\
\hline OLOS & 4.66 & 10.52 & 10.11 & 8.74 & -6.15 & 10.59 \\
\hline
\end{tabular}

The results demonstrate that the propagation characteristics are rather different between $V$ and $H$ polarized paths: $H$ polarized paths suffer more from depolarization than $V$ polarized paths. As shown, the power imbalance between $\left\langle\mathrm{XPD}_{H}^{p}\right\rangle$ and $\left\langle\mathrm{XPD}_{V}^{p}\right\rangle$ contrasts greatly with the averaged XPD computed directly from the measured channels (Table III from section III-B). For specular paths with low elevation (i.e., nearly parallel to azimuthal plane), the $H$ polarization will undergo more depolarization than the $V$ polarization because of the Brewster angle effect [22], [23], [13]. Conversely, for specular paths with high elevation, the Brewster angle effect will cause $V$ polarized paths to depolarize more than $H$ polarized paths. The paths with high elevation in the latter case are commonly those that reflect off the high ceiling. These paths are characterized by a large path length and thus low power. One can thus argue that the XPD for $V$ polarized paths is generally higher than for $H$ polarized paths because $V$ depolarization occurs more frequently for insignificant lowpower paths. This leads us to conclude that buildings with high ceilings, such as industrial halls, favor $V$ polarization over $H$ polarization.

Figure 9 presents a more detailed statistical study of the SMC XPD as a function of distance for LOS and OLOS scenarios. The LOS component has been removed to avoid any strong bias as it does not suffer from depolarization mechanisms. Boxplots were used to assess the XPD distribution of the polarized SMC. For each blue box, the central red marker is the median and the edges of the box extend to the $25^{t h}$ and $75^{t h}$ percentiles. For the sake of comparison, the averaged XPD of the measured channel and DMC are also depicted. Permutation tests on Spearman's rho concluded that the SMC and DMC XPDs do not correlate significantly with distance ( $p$-value $>0.14$ for $H / V$ and LOS/OLOS). The only exception was the DMC XPD for $H$ polarized waves in OLOS conditions (Fig. 9(c); Spearman's rho equalled -0.52 and was found significant at a $p$-value of 0.03). However, it appears that SMC and DMC XPD do generally decrease with distance showing that depolarization increases with distance (the same trend is found for the large halls in [13]). Nevertheless, the depolarization mechanim is very slow.

From the boxplot statistics, the median SMC XPD and DMC XPD values averaged over all positions are summarized in Table VI. First, larger de-embedded XPD values for DMC are found compared to values of $0 \mathrm{~dB}$ reported recently in indoor environments [21]. This shows that full depolarization is not achieved in this large volume environment. Again, this may be attributed to the position asymmetry of the arrays when measuring the environment due to the high ceiling but also due to the spatial distribution of the scatterers. Then, it appears the DMC depolarization is almost constant across LOS/OLOS and $H / V$ polarization with a mean value of $4.45 \mathrm{~dB}$ and standard deviation less than $3 \mathrm{~dB}$ for distances greater than $15 \mathrm{~m}$. The SMC and DMC XPD values for $H$ are very similar (5.72 vs. 5.29 for LOS and 6.11 vs. 6.84 for OLOS) highlighting the fact that they are both affected by the same propagation effects. Conversely, a larger depolarization is observed for $V$ polarized DMC compared to $V$ polarized SMC (11.53 vs. 5.17 for LOS and 9.98 vs. 4.16 for OLOS). Hence, the depolarization for $V$ channels is strongly caused by DMC propagation mechanisms.

Finally, the results highlight the fact that the polarimetric SMC and DMC obtained with the de-embedding approach provide a deeper physical comprehension of the radio channel propagation mechanisms compared to the classical one (section III-B) and should be applied in future studies.

TABLE VI

Averaged Median XPD For SMC \& Averaged XPD FOR DMC (IN $d B)$

\begin{tabular}{|c|c|c|c|c|}
\cline { 2 - 5 } \multicolumn{1}{c|}{} & \multicolumn{2}{c|}{ SMC } & \multicolumn{2}{c|}{ DMC } \\
\cline { 2 - 5 } & $\left\langle\mathrm{XPD}_{H}^{p}\right\rangle_{0.5}$ & $\left\langle\mathrm{XPD}_{V}^{p}\right\rangle_{0.5}$ & $\left\langle\mathrm{XPD}_{H}\right\rangle$ & $\left\langle\mathrm{XPD}_{V}\right\rangle$ \\
\hline \hline LOS & 5.72 & 11.53 & 5.29 & 5.17 \\
\hline OLOS & 6.11 & 9.98 & 6.84 & 4.16 \\
\hline
\end{tabular}

\section{DMC Reverberation Time}

Finally, the polarimetric DMC reverberation time $\tau_{r}^{X Y}$ of the environment was computed. The reverberation time is computed from the decay slope of the DMC by:

$$
\tau_{r}^{X Y}=\frac{B W}{\beta_{d}^{X Y}},
$$

where $B W$ is the measurement bandwidth in Hertz. Figure 10 presents $\tau_{r}^{X Y}$ (in ns) as a function of distance for 


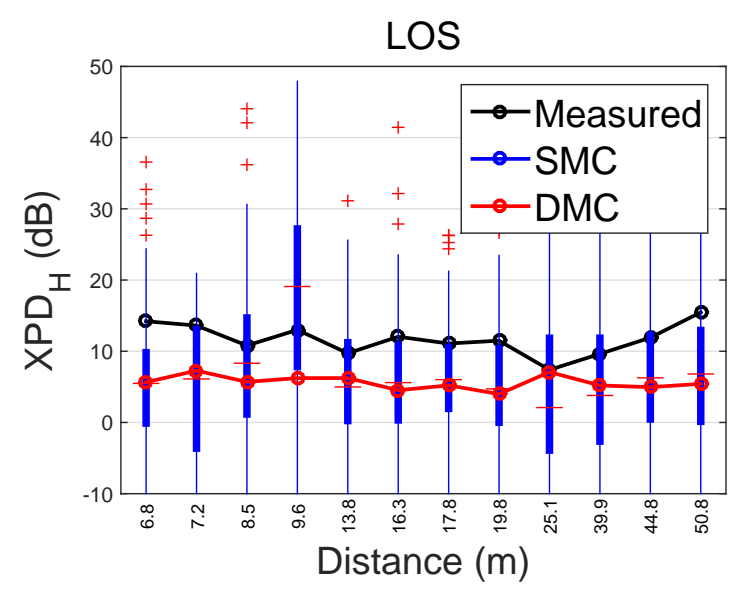

(a)

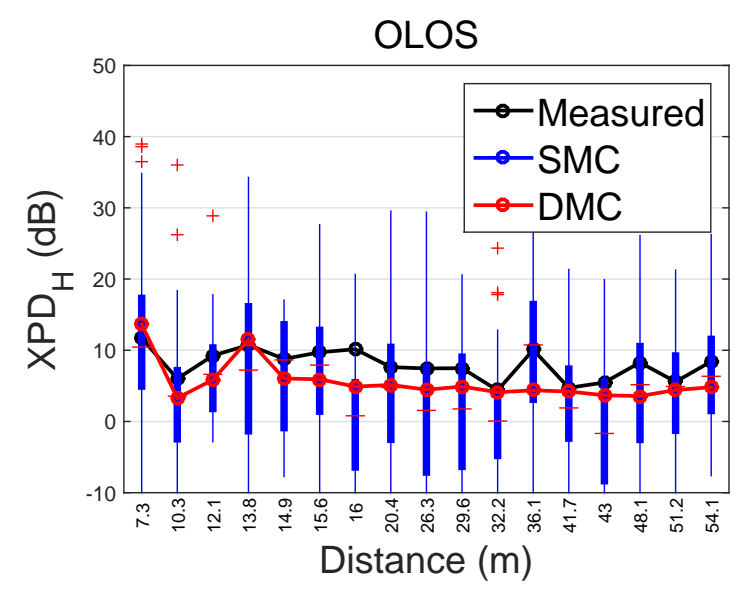

(c)

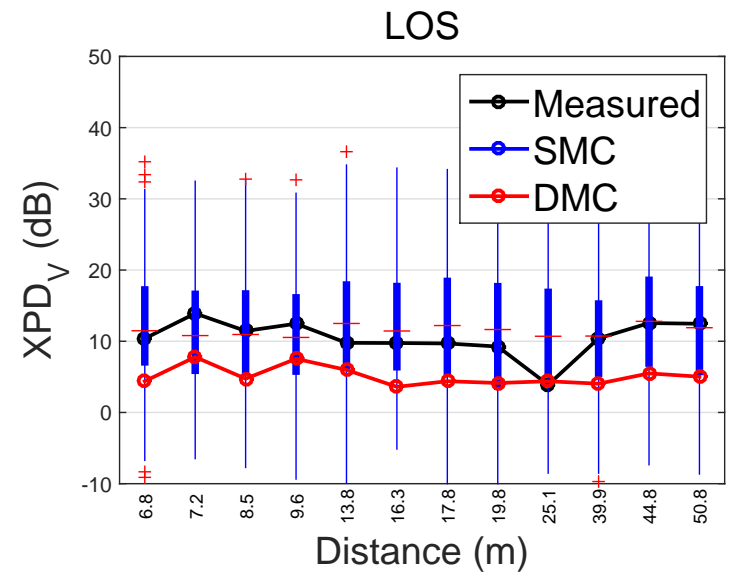

(b)

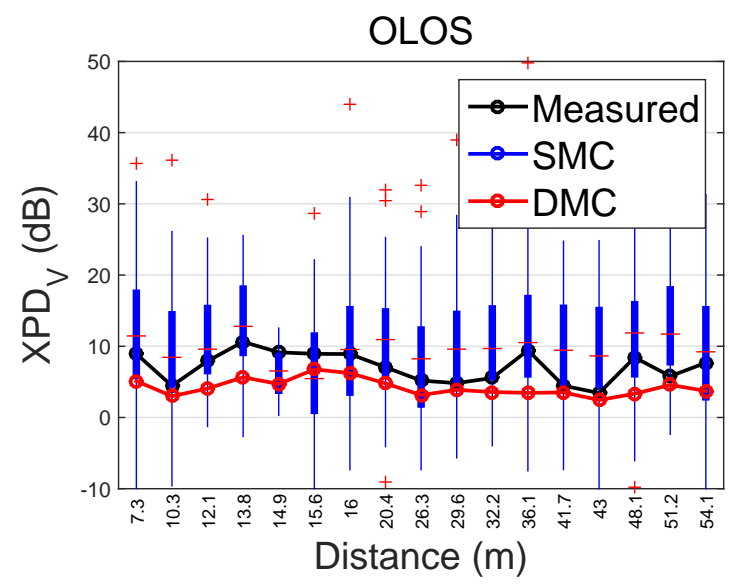

(d)

Fig. 9. Measured, de-embedded SMC, and DMC XPD values (in $\mathrm{dB}$ ) as a function of distance (in m) for $H$ (left column) and $V$ (right column) and for LOS (top row) and OLOS (bottom row) scenarios. Boxplots statistics were computed for the SMC. For each blue blox, the central red mark is the median, the edges of the box are the $25^{t h}$ and $75^{t h}$ percentiles, the whiskers extend to the most extreme data points not considered outliers (red crosses).

LOS and OLOS scenarios. The results demonstrate that the reverberation time is almost constant across polarization and distance. It appears to be slightly dependent on distance when the Tx - Rx distance is within 15 meters. On the other hand, no dependence can be found for distances greater than $15 \mathrm{~m}$ and also the difference between LOS and OLOS is found to be small enough to be neglected. A mean value of $75 \mathrm{~ns}$ (70 ns) is found for LOS (OLOS) for distances beyond 15 meters and mean value of $70 \mathrm{~ns}$ ( $66 \mathrm{~ns}$ ) if all positions are taken into account. The standard deviation is $4.3 \mathrm{~ns}$ and $5 \mathrm{~ns}$ for LOS and OLOS, respectively. Therefore, one can conclude that, despite the large volume of the environment and high ceiling, DMC follows the Room Electromagnetics theory [4]. Hence, simple DMC models can be used to design more advanced channel models as proposed in [10].

\section{CONCLUSION}

The joint polarization characteristics of SMC and DMC have been investigated in a large industrial hall, based on frequency-domain channel sounding experiments at $1.3 \mathrm{GHz}$ with $22 \mathrm{MHz}$ bandwidth. 29 positions were measured under

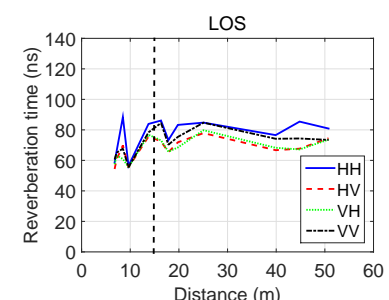

(a)

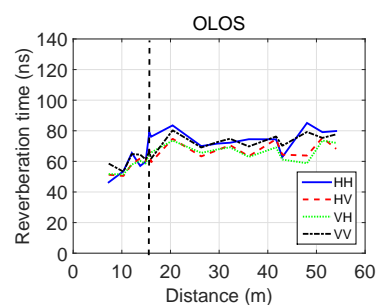

(b)
Fig. 10. Polarimetric reverberation time (in ns) as a function of distance (in $\mathrm{m}$ ) for LOS (left) and OLOS (right) scenarios. The dotted vertical line indicates the $15 \mathrm{~m}$ distance.

either strong LOS or OLOS scenarios. The full-polarimetric SMC and DMC were estimated with RiMAX by deembedding the polarimetric radiating patterns of the dualpolarized measurement antennas. The computed polarimetric delay and angular spread distributions were physically related to the fading mechanisms as well as the Tx/Rx location in the environment. Measured XPD values for the $H$ and $V$ polarization are balanced for both LOS and OLOS scenarios 
and are shown to be in agreement with smaller volume indoor environments. In comparison, strong de-embedded SMC depolarization is observed for the $H$ polarization in OLOS scenarios. On the other hand, DMC depolarization is observed to be weaker compared to previous studies in nonindustrial environments due to the high ceiling but nearly constant across all shadowing conditions, polarizations, and distances. The results also show that the co-polar (crosspolar) DMC power to total channel power ratio is equal to $15 \%(40 \%)$ for LOS and $40 \%(60 \%)$ for OLOS and that this ratio does not correlate significantly with transmitter-receiver distance. Finally, a mean reverberation time of $70 \mathrm{~ns}$ with low standard deviation validates the room electromagnetics theory for transmitter-receiver distances larger than $15 \mathrm{~m}$ with no significant difference between polarized subchannels.

\section{ACKNOWLEDGMENT}

E. Tanghe is a Post-Doctoral Fellow of the FWO-V (Research Foundation - Flanders). This work was financially supported by the INTERREG IVa project 1.1.7 WiSE and the project IAP BESTCOM, BElgian network on STochastic modelling, analysis, design and optimization of COMmunication systems. This research was also partly funded by the Fund for Scientific Research - Flanders (FWO-V, Belgium) project G.0325.11N.

\section{REFERENCES}

[1] A. Richter, "Estimation of radio channel parameters: Models and algorithms," Ph.D. dissertation, Technische Universität Ilmenau, Fakültat für Elektrotechnik und Informationstechnik, Ilmenau, DE, 2005. [Online]. Available: http://www.db-thueringen.de/servlets/DerivateServlet/Derivate7407/ilm1-2005000111.pdf

[2] C. Holloway, M. Cotton, and P. McKenna, "A model for predicting the power delay profile characteristics inside a room," IEEE Trans. Veh. Commun., vol. 48, no. 4, pp. 1110-1120, Jul 1999.

[3] R. Rudd, "Statistical prediction of indoor radio channel impulse response," Ph.D. dissertation, Univ. Surrey, Surrey, U.K., 2007.

[4] J. Andersen, J. Nielsen, G. Pedersen, G. Bauch, and M. Herdin, "Room electromagnetics," IEEE Trans. Antennas Propag., vol. 49, no. 2, pp. 27-33, April 2007.

[5] J. Nielsen, J. Andersen, G. Pedersen, and M. Pelosi, "On polarization and frequency dependence of diffuse indoor propagation," in Vehicular Technology Conference (VTC Fall), 2011 IEEE, Sept 2011, pp. 1-5.

[6] A. Bamba, W. Joseph, E. Tanghe, G. Vermeeren, and L. Martens, "Circuit model for diffuse multipath and electromagnetic absorption prediction in rooms," IEEE Trans. Antennas Propag., vol. 61, no. 6, pp. 3292-3301, June 2013.

[7] A. Bamba, W. Joseph, G. Vermeeren, E. Tanghe, D. P. Gaillot, J. B. Andersen, J. d. Nielsen, M. Lienard, and L. Martens, "Validation of experimental whole-body sar assessment method in a complex indoor environment," Bioelectromagnetics, vol. 34, no. 2, pp. 122-132, February 2013 .

[8] D. A. Hill, Electromagnetic Fields in Cavities: Deterministic and Statistical Theories, ser. IEEE Press Series on Electromagnetic Wave Theory. Piscataway, NJ: Wiley/IEEE Press, 2009.

[9] O. Delangre, "Radio channel characterization and models in reverberating rooms," Ph.D. dissertation, University of Lille1, Villeneuve d'Ascq, FR and Universit Libre de Bruxelles, Brussels, BE, 2008.

[10] G. Steinbock, T. Pedersen, B. Fleury, W. Wang, and R. Raulefs, "Distance dependent model for the delay power spectrum of in-room radio channels," IEEE Trans. Antennas Propag., vol. 61, no. 8, pp. 43274340, Aug 2013.

[11] M. Landmann, "Limitations of experimental channel characterisation," $\mathrm{Ph} . \mathrm{D}$. dissertation, Technische Universität Ilmenau, Fakültat für Elektrotechnik und Informationstechnik, Ilmenau, DE, 2008. [Online]. Available: http://www.db-thueringen.de/servlets/DerivateServlet/Derivate15967/ilm1-2008000090.pdf
[12] T. Rappaport, Wireless Communications: Principles and Practice, 2nd ed. Upper Saddle River, NJ, USA: Prentice Hall PTR, 2001.

[13] M. Kim, Y. Konishi, Y. Chang, and J.-I. Takada, "Large scale parameters and double-directional characterization of indoor wideband radio multipath channels at $11 \mathrm{GHz}$," Antennas and Propagation, IEEE Transactions on, vol. 62, no. 1, pp. 430-441, Jan 2014.

[14] J. Poutanen, J. Salmi, K. Haneda, V. Kolmonen, and P. Vainikainen, "Angular and shadowing characteristics of dense multipath components in indoor radio channels," IEEE Trans. Antennas Propag., vol. 59, no. 1, pp. 245-256, 2011.

[15] F. Mani, F. Quitin, and C. Oestges, "Directional spreads of dense multipath components in indoor environments: Experimental validation of a ray-tracing approach," IEEE Trans. Antennas Propag., vol. 60, no. 7, pp. 3389-3396, 2012.

[16] A. Richter, J. Salmi, and V. Koivunen, "Distributed scattering in radio channels and its contribution to MIMO channel capacity," in Antennas and Propagation, 2006. EuCAP 2006. First European Conference on, Nov 2006, pp. 1-7.

[17] F. Quitin, C. Oestges, F. Horlin, and P. De Doncker, "Diffuse multipath component characterization for indoor MIMO channels," in Antennas and Propagation (EuCAP), 2010 Proceedings of the Fourth European Conference on, April 2010, pp. 1-5.

[18] E. Tanghe, D. Gaillot, M. Lienard, L. Martens, and W. Joseph, "Experimental analysis of dense multipath components in an industrial environment," IEEE Trans. Antennas Propag., vol. 62, no. 7, pp. 37973805, July 2014.

[19] V. Anreddy and M.-A. Ingram, "Capacity of measured ricean and rayleigh indoor MIMO channels at $2.4 \mathrm{GHz}$ with polarization and spatial diversity," in Wireless Communications and Networking Conference, 2006. WCNC 2006. IEEE, vol. 2, April 2006, pp. 946-951.

[20] J.-M. Molina-García-Pardo, J.-V. Rodríguez, and L. Juan-Llácer, "Polarized indoor MIMO channel measurements at $2.45 \mathrm{GHz}$," IEEE Trans. Antennas Propag., vol. 56, no. 12, pp. 3818-3828, Dec 2008.

[21] E. Vitucci, F. Mani, C. Oestges, and V. Degli-Esposti, "Analysis and modeling of the polarization characteristics of diffuse scattering in indoor and outdoor radio propagation," in Applied Electromagnetics and Communications (ICECom), 2013 21st International Conference on, Oct 2013, pp. 1-5.

[22] P. Kyritsi and D. Cox, "Propagation characteristics of horizontally and vertically polarized electric fields in an indoor environment: simple model and results," in Vehicular Technology Conference, 2001. VTC 2001 Fall. IEEE VTS 54th, vol. 3, 2001, pp. 1422-1426 vol.3.

[23] Y. Lostanlen and T. Tenoux, "Electromagnetic wave depolarization behaviour at different frequencies for various dielectric materials," in Antennas and Propagation, 2007. EuCAP 2007. The Second European Conference on, Nov 2007, pp. 1-6. 